\title{
The Frame(s) Problem and the Physical and Emotional Basis of Human Cognition
}

\author{
Carlos Acosta \\ Independent Cognitive Scholar \\ San Luis Obispo, California, USA
}

\begin{abstract}
This paper focuses on the intriguing relationship between mathematics and physical phenomena, arguing that the brain uses a single framework to order, arrange, and process basic information into more complex thought and knowledge. It is proposed that multiple incremental permutations of this single format eventually give rise to all abstract thought. The main thesis overcomes the epistemological complexities of the Frame(s) Problem by asserting that the primal frame of reference - from which all conscious thought ultimately emerges - is essentially a chaotic representation of the four dimensional properties of existence plus the genetically derived causal objective, and the embodied emotional behaviours that even the lowliest cognitive organisms are born with, and which they automatically express as they struggle to exist within an ever-changing and often hostile environment.
\end{abstract}

Keywords: frame of reference; schematic systems; a priori knowledge; finite regress; chaotic systems; feedback loops

\section{Introduction}

Natural philosophers have long recognized that mathematics describes the underlying nature of reality. In 1623, for example, Galileo declared:

Philosophy is written in this grand book, $[\ldots]$ which stands continually open to our gaze, but cannot be understood unless one first learns to comprehend the language and interpret the characters in which it is written. It is written in the language of mathematics, and its characters are triangles, circles, and other geometric figures without which it is humanly impossible to understand a single word of it; without these one is wandering in a dark labyrinth. ${ }^{1}$

Though scientists use mathematics to understand the workings of the cosmos, they do not, in fact, view mathematics itself as a true empirical science. Scientists and mathematicians alike tend to see mathematics as a rigorous deductive discipline distinct from the inductive reasoning of pure science. The difference is not just in the semantics. A deductive analysis begins from a diverse set of generally accepted principles assumed to be true. In mathematics, these first principles are axioms.

\footnotetext{
${ }^{1}$ Il Saggiatore, (Rome, 1623) The Assayer, in: The Controversy of the Comets of 1618 (University of Pennsylvania Press, 1960), pp. 183-4.
} 
From this beginning the deductive process moves through a series of logical steps, via the rules of inference, to a single specific conclusion. Once a deductive conclusion has been arrived at, it is thereafter always assumed to be true until or unless any of the logical steps and/or the principles upon which they are based can be refuted.

An inductive empirical investigation, on the other hand, starts by gathering information through the observation of a specific natural phenomenon. It then proceeds through a series of logical steps to a general explanation (i.e., a scientific theory) of the observed facts. Finally, in order to test its validity - that is to say, to confirm whether a given explanation of nature is true or false - the theory in question is empirically compared to the underlying reality it attempts to describe. Unlike a deductive conclusion, a scientific theory is never taken to be true unless it is in total agreement with observable reality. Therefore, scientific paradigms often change or are reinterpreted over time as new information concerning the true nature of physical existence comes to light.

Deductive thought moves from the general to the specific, while empirical reasoning proceeds from the specific to the general. Though outwardly deductive logic seems to be at odds with inductive empirical reasoning, deductive mathematical theorems readily lend themselves to the description of physical reality. No one is quite sure why this should be the case, but it leads to the harmonious recurring interplay between mathematics and existence described by the influential German mathematician David Hilbert.

In Hilbert's famous "Mathematical Problems" address, we learn that "[...] the first and oldest problems in every branch of mathematics spring from experience and are suggested by the world of external phenomena" (Hilbert, 1900). ${ }^{2}$ From this simple beginning, once started, the human mind internally continues to deductively develop new mathematics on its own, largely independent of outside influences. Once developed, this new mathematics is frequently used to gain fresh insights into the nature and structure of physical phenomena. In turn, these insights lead to deeper questions concerning the true essence of reality which are often answerable only through a further advance in abstract mathematics. That is the quintessential meaning of Hilbert's (p.4) "[...] ever-recurring interplay between thought and experience."

Why should there be interplay between deductive thought and reality? Why does mathematics - this internal creation of the human mind - continually evolve to exactly describe the external web of reality surrounding us? In their book, The Non-Local Universe, mathematician Menas Kafatos and scientific historian Robert Nadeau (2001, p.102), raise essentially this same question in a slightly different context. They expressively ask, "Why is there a correspondence between physical theory and physical reality, or between the mind capable of conceiving and applying mathematical physics and the Cosmos itself?"

\footnotetext{
${ }^{2}$ Mathematical Problems, (Paris, 1900) in: Mathematical Developments Arising from Hilbert Problems, (1976): American Mathematical Society, p. 3. Reprinted from Bulletin of the American Mathematical Society 8 (July 1902), pp. 437-479. Originally published as Mathematische Probleme Vortage, gehalten auf dem internationalen Mathematike-Congress zu Paris 1900, Gött. Nachr. 1900, pp. 253-297, Vandenhoeck \& Ruprecht, Göttingen.
} 
In the mid-20th century, scientists, such as the eminent physicist and mathematician Eugene Paul Wigner (1902-1992), believed that the unreasonable effectiveness of mathematics in describing the cosmos was "[...] something bordering on mysterious" (1960, p. 2). However, in the first years of the 21 st century cognitive scholars such as George Lakoff and Rafael Núñez began espousing a more pragmatic approach. In their book, Where Mathematics Comes From (2000, p. 3), the two researchers proclaim:

[...] that whatever "fit" there is between mathematics and the world occurs in the minds of the scientists who have observed the world closely, learned the appropriate mathematics well (or invented it), and fit them together, (often effectively) using their all-too human minds and brains.

They additionally assert that,

It is sometimes assumed that the effectiveness of mathematics as a scientific tool shows that mathematics exists in the structure of the physical universe. This, of course, is not a scientific argument with any empirical scientific basis.

This author concurs with much of the research material presented by Lakoff and Núñez, but not these two pronouncements. Both statements are true but somewhat limited in their scope. The former correctly depicts the external circumstances, but does not adequately explain why there should be any internal correspondence between mathematics and reality within the minds of the scientists in the first place. Nor does the latter pronouncement explicitly preclude the possibility that a scientific theory, along the lines suggested, may someday be forthcoming.

The question, so eloquently posed by Kafatos and Nadeau, remains a bewildering conundrum. But this mysterious correlation between mathematics and reality may be a piece to a larger puzzle - a clue to the deepest underlying physiological relationship between the human mind and external existence. This essay examines the fascinating correspondence between the mind and physical phenomena by putting forward a simple, frames-based examination of the fundamental structure of conscious thought in relation to mathematics, and to the following key points:

- That non-intuitive physical conditions seem to have been genetically imposed on us by external reality,

- That mathematical objects, though imaginary, may have real initial properties,

- $\quad$ That mathematics may be a modified mental model of reality, and

- $\quad$ That the brain may generate a series of embedded contextual reasoning systems that cumulatively produces conscious thought. 


\section{Problems with Game Theory}

I propose to launch this investigation into the physiological basis of mathematics and the underlying structure of complex thought by inductively exploring the foundations of game theory. The broadly defined field of game theory provides useful insights into fundamental concepts, not only of mathematics but also of conscious thought itself. For, as soon as a cognitive organism reacts to its environment it is often plunged, like it or not, into life-and-death scenarios very much akin to game-playing. Gaming, one might argue, mirrors the survival basis of thought. One could even see nature represented in games and vice versa.

Game theory is the branch of mathematics and logic that investigates the actions of game players. Game playing is complex thought in microcosm, for it involves decisionmaking. Valid decision-making necessitates the prior development of an intricate system of knowledge to determine the most appropriate actions and behaviours that thinkers (in this case, the players) must undertake in order to resolve specific and often highly abstract game-related problems.

No complex system of thought may be generated, however, without first confronting the simple contextual arrangement of information within which our environmental stimuli and actions are played out. Inductively examining the broadest contextual structure of all games, and paying special attention as to how the mind may draw mathematical meaning from similar informational constructs, provides us with a first step toward understanding how the brain orders, arranges, and processes basic information into more complex thought and knowledge.

Curiously, none of the sources on game theory consulted for this paper describes the underlying structure of any game. Most theorists simply define games as "conflicts involving gains and losses between two or more opponents who follow formal rules" or another similarly broad definition. The writer then typically moves on to examine specific mathematical relationships between the players.

It seems likely that this rather casual approach to defining a game or games is due to the fact that we all possess some intuitive grasp or innate perception of what games are. This presumptive sense of the general nature of all games might disincline us from looking further into their basic structure. It may well be that our inability to look beyond the stated definition of a game has tremendous ramifications when it comes to unraveling the mysterious effectiveness of mathematics in describing the workings of the cosmos.

From the perspective of game theory, the mathematical definition of a game should be broad as it must allow for all the exceedingly diverse human interactions that may correctly be considered games. For this reason, the standard definition of games as "conflicts involving gains and losses between two or more opponents who follow formal rules" has thus far been accepted.

However, there are three distinct problems with this definition. First, not all games are conflicts; a more appropriate terminology would be "interactions between two or more individuals". Second, this description does not address the underlying structure of any game. Third, it appears that the "formal rules" aspect of this definition has been too broadly applied. 
Although there are broad variations in outward appearance from game to game, all games share the same fundamental structure. On close examination it can be seen that all games, whether board games, parlour games, or sports, possess the same five defining characteristics, or Five Elements:

1. Field

2. Elements

3. Rules

4. Objectives

5. Feedback Loop

- A field may be defined as a static, non-moving bounded area of space, in or on which the elements move. (1)

- Elements are objects that move, or are caused to move, along or within a field. (2)

- Rules, i.e., the action component, are descriptions of how the elements are positioned in space, and move or are caused to move in relation to the field and relative to each other. (3)

- The objective is the underlying rationale that initiates an action resulting in a gain or a loss, or which can be perceived in the more general positive and negative terms of a concept and its opposite. (4)

- The feedback loop is any action or reaction to the prior movement of elements within the field over time. (5) (Note: when an object changes position along or within the field, we contextually perceive this movement in terms of a span or duration of time. Accordingly, the "fourth dimension" of the external physical universe is implicitly understood to be the sixth operational element of the frame of reference of any game. $)^{3}$

All games share this modified spatiotemporal configuration. The outward expression of each criterion may differ from game to game, but their underlying commonality remains. In fact, none of the above-mentioned essential characteristics is new. They have always inherently described the general form of any game. In the past, however, it was incorrectly assumed that rules alone govern every descriptive aspect of a game. As a result, all the perceptual classifications were inappropriately grouped together under this one-dimensional catch-all descriptive heading. In the strictest sense, the five core properties of all games, plus time, should not be lumped together under such an allencompassing designation as "rules," but instead, each principal game element should

\footnotetext{
${ }^{3}$ When physicists describe time as the fourth-dimension, they have in mind the three spatial dimensions of height, length, and width combined with time. By contrast, the term "fourth dimension" as utilized in this paper is meant to convey the four macro properties of space, matter, motion, and time, also with time as the fourth but distinct element in the series. This convention is intended to emphasize the temporal dimension in a manner that is more in keeping with the way it is subjectively perceived.
} 
properly be considered as a distinct contextual category of information within a single frame of reference.

\section{Contextual Schematic Meaning}

This holistic description of the underlying structure of all games accurately portrays the physical and mental parameters within which the tactics and strategy of any game develop. Moreover, it allows us to recognize that, for games at least, the core of meaning is contextual, and that all game-specific systems of knowledge, no matter how divergent, grow and develop within the same conceptual framework.

The five contextual categories and time may all be seen to be of equal and vital importance. They form a single interconnecting and interacting continuum of basic information within which a more complex game-specific system of thought germinates. If any one of these six data categories were eliminated from this cognitive framework, the resulting interacting thought system simply would not develop.

In order to support this particular inductive observation, the reader is invited to conduct the following thought experiment. Think of a game; any game at all. Now completely eliminate any one of the information classifications and see if that game can still be played. One may be inclined to think that games such as dominoes, cards, or tic-tac-toe are exceptions, as they are not played on physically defined fields but - on tables, paper, or bare ground. Tables or bare ground still comply with my previous definition of a playing field, given that the contextual action takes place in or on a non-moving bounded area of space limited to how far the player can reach. Even a card game such as solitaire requires a differentiated "field" where "elements," i.e., cards, can be displayed and laid out in random or sequential relationships to one another. In a game as minimal as paperrock-scissors, the bounded field is the space between the two players where the "objects" are deployed as opposed to behind one of the player's back or in his or her pocket.

Furthermore, it appears that the system of thought associated with this contextual framework becomes directly dependent on the perceptual characteristics of the various information classifications. Changing the expression (i.e., the specific details) of any one of the six criteria (but not necessarily the essential property of the element itself) dramatically changes the content of this embedded thought system.

Time and the five components of the contextual framework set the basic parameters or initial conditions for any game. On a fundamental level, they describe how the game is to be played, and set forth the what, when, where, how, and why of the game. Significantly, they do not explain tactics and strategy. The strategy and tactics of any game emerge as a complex system of interrelated knowledge or schemas within the feedback loop of the contextual framework. The feedback loop evolves over time. It is a criterion of the complete frame of reference of a game, for knowledge cannot develop without it, but strictly speaking, it is not structurally part of the frame of reference itself but rather embedded in it.

A schema, or the strategy developed within the feedback loop, is a heuristic plan of action and reaction that anticipates future events based on past remembered experiences in relation to present circumstances. Schemas embody the principle of induction, which takes as a given that if a specific physical phenomenon or complex situation has occurred under certain conditions in the past, it will reoccur under those same conditions in the 
future. Schemas adhere to a simple "if-then" format. That is to say, if this happens, then do that, or when that happens, then do this. Over time, new more intricate schemas develop from simple ones; they double up on themselves, so to speak, and grow in complexity through an action-reaction feedback loop of trial and error.

Notably, the schemas of any game also describe how a game is to be played, but on a higher order of perceptual complexity. As with the more intricate strategizing that allows us to perceive an abstract threat on a chessboard, schemas are inherently more complex patterns of information; from their very inception they automatically incorporate the basic spatiotemporal arrangement of information for the particular frame of reference from which they spring. No matter how complex the tactical and strategic schemas of any game may become, they are endowed with meaning only in relation to their original contextual framework.

\section{The Frame(s) Problem}

Within the confines of a game, one can see that the elaborate accumulation of tactical and strategic knowledge (i.e., schemas) is contextual. Is this simple insight gamespecific, or can one expand this inductive observation to encompass all meaning and all knowledge? This paper argues that such an extension is warranted, and that all meaning and knowledge emerge as contextual schemata within distinct frames of reference. ${ }^{4}$ However, it must be noted that whenever one attempts such a straightforward inductive leap, one risks sinking into an epistemological quagmire. The first epistemological problem we encounter is inherent in all inductive reasoning, namely: we may never be certain that the phenomena we inductively observe in a small class of objects can be extended to all classes.

Even if we sidestep this first problem for the sake of argument or experimentation, it only leads directly to the puzzle that is the Frame(s) Problem. This problem arises from our insistence that all meaning and all knowledge emerge within a contextual framework. For, if we hold fast to this original inference that all knowledge and meaning develop as a system of embedded schemas within a frame of reference, we are then faced with the problem of how any (initial/ultimate) frame of reference acquires meaning and structure prior to the development of a given system of contextual thought.

We may at first attempt to answer this question by regressively postulating that the concepts that confer structure and prior meaning to our original frame of reference are simply part of a different system of knowledge derived from a second frame of reference. By taking this position, one immediately encounters the dilemma at the heart of the Frame(s) Problem. For, if we take this view, then the original question has not been answered, merely transferred to the second frame of reference. We may attempt to answer the original question as it pertains to the second frame of reference by creating a third frame of reference, but the problem is again transferred, this time to the third frame of reference. One can create a fourth frame and a fifth, and so on indefinitely, but the

\footnotetext{
${ }^{4}$ In its purest form, the paradigm of conceptual emergence simply holds that new and unexpected cognitive properties and processes develop from older more basic ones. For a more detailed empirical treatment of contextual emergence see (Atmanspacher, 2007), (Atmanspacher and Graben, 2007), and (Bishop and Atmanspacher, 2006).
} 
complex system of knowledge that arises from this infinite regression can never answer the original question.

\subsection{A Priori Knowledge}

Clearly, the Frame(s) Problem arises out of our attempts to empirically comprehend how any primal frame of reference is originally endowed with prior meaning and structure. At first glance it would seem as though this problem is impossible to answer; yet we may be able to shed rational light on this subject by examining that intriguing and closely associated phenomenon known as a priori knowledge.

"A priori" denotes a class of knowledge independent of physical experience. The philosopher Immanuel Kant (1724 - 1804) employed this term extensively in his Critique of Pure Reason (1781). ${ }^{5}$ According to him, a priori knowledge may be thought of as internalized fundamental information that we all somehow possess. This knowledge does not correlate with physical phenomena directly, but exists nonetheless as valid information about reality and is necessary in order to make rational judgments concerning new experiences and perceptions. Kant argued that the axioms and any new mathematical theorems are built-up from our a priori, i.e., intuitive knowledge of the external physical world. He also maintained that this intuitive information exists in four distinct categories which, he said, organize the world of material experience.

These four categories are: quantity, quality, relation, and modality. Kant further divided each of these major categories into three subgroups. He divided the "quantity" category into the subgroups of unity, plurality, and totality. He divided the category of quality into the three subgroups of reality, negation, and limitation. He divided the "relation" category into substance-and-accident, cause-and-effect, and reciprocity. Lastly, he divided the category of modality into possibility, existence, and necessity.

In mathematics, a priori knowledge may be observed in the simple commutative law of addition, for example: $(a+b)=(b+a)$. The statement that $(b+a)=(a+b)$ is selfevident. The internalized subconscious system of prior logic that allows us to intuitively recognize that order does not matter in addition is what Kant termed a priori knowledge. As illustrated in the closely related book, Prolegomena to Any Future Metaphysics (Kant, 1783), in regard to the axioms of mathematics Kant wrote:

$[\ldots] \mathrm{a}=\mathrm{a}$, the whole is equal to itself, or $(\mathrm{a}+\mathrm{b})>\mathrm{a}$, i.e., the whole is greater than its part. And indeed even these, although they are valid from concepts alone, are admitted into mathematics only because they can be exhibited in intuition. ${ }^{6}$

In Kant's philosophy, new information about external existence is gained through the evaluation of propositions - which he termed "judgments" - that are either analytic or synthetic. Analytic propositions can be thought of as information that maybe arrived at

\footnotetext{
${ }^{5}$ Kant's Critique of Pure Reason was first published in 1781. A revised second edition followed six years later. The latter version is widely considered the more accessible of the two. This paper relies primarily on an English translation of the second edition.

${ }^{6}$ Prolegomena to Any Future Metaphysics, Cambridge Texts in The History of Philosophy (Cambridge University Press 2004), p. 19.
} 
through logic alone, while synthetic propositions are formalized through contemplative observation. In this sense, synthetic knowledge is empirical in nature, but Kant further defined knowledge as either being a posteriori or a priori. A posteriori information is gained through prior sensory experience and is therefore subject to interpretation, but $a$ priori knowledge is independent of experience. It is knowledge that does not contradict itself or anything else that is true, and that is based on elemental intuitions. Kant steadfastly maintained that new mathematical information is entirely synthetic a priori and not strictly analytical because it depends on the pure intuitions of the elements of space and time. According to Garth Kemerling, "[...] Kant argued in the

"Transcendental Aesthetic" of the First Critique, (that space and time) are the pure forms of sensible intuition under which we perceive what we do." 7

A priori knowledge exists as human intuitions - mysterious but consistent internal patterns of information - that we all possess. This internalized knowledge is necessary in order to judge and evaluate new experiences and new perceptions. The existence of $a$ priori knowledge is not in dispute; most psychologists and other cognitive researchers agree that it exists and is valid; they are, however, rather uncomfortable with Kant's philosophical description of it. For how is it possible for humans to somehow already (i.e., a priori) possess knowledge that enables us to comprehend new information before this new information is even discovered? Consequently, the central question one must address when investigating where mathematics comes from is the same one explored by Immanuel Kant over 200 years ago, namely: what engenders our mysterious intuitions about axioms and any new mathematics? In other words, what is the true nature of $a$ priori knowledge? Where does it come from? How is it generated? Provided that we can develop an empirically consistent explanation for the phenomenon, a priori knowledge may provide us with a partial answer as to how any contextual system of higher-order perception acquires the capacity to understand and manipulate new information.

\section{Finite Regress}

From our brief study of games, it is evident that elaborate game-specific tactical and strategic knowledge develops within, and only has conscious meaning in relation to, a simpler contextual framework. We may expand this observation by taking the position that all knowledge emerges as an interconnected system of complex schemata within multiple hierarchical frames of reference. If we do so, however, we must rationally explain how the original contextual framework acquired its own meaning and structure prior to the development of an embedded thought system. Attempting to solve this philosophical riddle, through an infinite regression of ever more complex frames of reference, leads directly to the puzzle of the Frame(s) Problem. A "finite regress" of less and less complex frames of reference seems a viable alternative, and it may even provide the long-sought solution to this epistemological enigma.

The research of the renowned developmental psychologist Jean Piaget indicates that such a finite regress may indeed be the answer, for Piaget was of the opinion that human thought logically develops from a process of inclusion (i.e., nesting) of lower, less-

\footnotetext{
${ }^{7}$ Garth Kemerling, 'Kant: Synthetic A Priori Judgments, ' http://www.philosophypages.com/hy/5f.htm Accessed 25 May 2006.
} 
powerful embedded structures superseding one another into higher and more powerful ones. $^{8}$

If one takes Piaget's phrase "embedded structures" to mean the frames of reference previously studied and their accompanying complex reasoning systems, and if the lower less powerful embedded structures are taken to be less complex frames of reference and less complex associated systems of thought that are imbued with meaning by a priori knowledge, then one may logically infer that conscious thought may arise out of a hierarchical descending series of iterated and interlocking frames of reference and their associated reasoning systems. Keeping in mind that the higher-order schematic reasoning systems are always more complex than their lower-order counterparts, and that the entire process is NOT an infinite regress, i.e., that it should logically stop after a finite number of levels, we see that we must eventually arrive at a primal frame of reference.

However, we still face the original epistemological conundrum, because this first frame of reference would very likely be devoid of any meaning as we have come to understand it. Nonetheless, it would be the ultimate framework from which all succeeding frames of reference emerge and from which all conscious thought and knowledge eventually develops. Questions would still remain as to how this first frame of reference came to be formed, what primal organizational structure it possesses, and what sort of initial meaning it has. But if these questions could be answered, then this first frame of reference could well be shown to be the original manifestation of a priori knowledge and the true contextual core of all meaning.

This paper proposes the existence of a primal a priori frame of reference, the fountainhead from which all succeeding frames of reference emerge, and from which all conscious thought and knowledge spring. The problem remaining is that a complete empirical explanation for this hypothetical original frame of reference, for a priori knowledge and thereby for all conscious thought, has yet to be developed.

The process of piecing together such a broad synthesis of ideas will be discussed in later papers, but for the moment we will confine our examination exclusively to the physiological foundations of mathematics with regard to the underlying elemental structure of all games and to the following key points:

- That non-intuitive physical conditions seem to be genetically imposed on us by external reality,

- That mathematical objects, though imaginary, may have real initial properties,

- That mathematics may be a modified mental model of reality, and

- That the brain may generate a series of embedded contextual reasoning systems that cumulatively produce conscious thought.

A clue that a connection exists between mathematics and the fundamental structure of all games - and by extension to all physical reality - may be found in the analogous correlation between the development of the tactics and strategy of a game and the development of mathematical theorems describing the universe. Over time, the tactics

\footnotetext{
${ }^{8}$ Jean Piaget Society: http://www.piaget.org/aboutPiaget.html Accessed 02 April 2005.
} 
and strategies of game-players tend to evolve into an optimum description of how a game is best played. This is comparable to the development of mathematics. Over time, our mathematical theorems, in conjunction with physical science, seemed to have similarly evolved to provide the best available description of how the universe functions.

\section{Number Theory}

Returning to the primary discourse, we may observe that mathematics adheres to the previous assertion that all thought and knowledge develops within a contextual framework. Upon close examination, we analogously see that number theory seems to correspond exactly to the frame of reference of a game. (Note: in subsequent articles, I endeavor to demonstrate that all branches of mathematics, not just number theory, can be understood within the same contextual framework). ${ }^{9}$ For the moment, however, we will concentrate solely on this one branch of mathematics.

In general terms, the "field" of number theory encompasses the entire frame of reference of a game with its five distinct categories of information:

\section{Field \\ 2. Elements \\ 3. Rules \\ 4. Objectives \\ 5. Feedback Loop}

More specifically, the Field equates to the set of real numbers. The Elements contained in this set include the positive and negative whole numbers plus zero. The Rules criterion corresponds to the rules by which numbers are manipulated - in this case, the axioms of number theory. For thousands of years, these axioms have been taken to be self-evident statements about the manipulation of numbers and have been assumed to be true without proof. The axioms of number theory are augmented by the rules of logical inference from which theorems are derived and by the arithmetical operations of addition, subtraction, multiplication and division. Objectives: In all mathematics, including number theory, the overall objective is to prove a proposition. A proposition is either true, (i.e., positive terms), false, (i.e., negative terms), or impossible. Impossibility can also be described in terms of true and false, for in mathematics a proposition is impossible if it can be proven that the proposition can neither be proven true nor false under the specific conditions of the given proposition. ${ }^{10}$

\footnotetext{
${ }^{9}$ This idea is not new. The following quote: "Mathematics is a game played with meaningless marks on paper," is a good case in point. The famous citation has been variously attributed to David Hilbert, but in actuality it is a corruption of a passage attacking his formalist ideals. The passage in question is part of the 1926 paper, Mathematical Logic, by the British mathematician F. P. Ramsey. In it Ramsey stated: "Mathematics proper is thus regarded [i.e. by the formalists] as a sort of game played with meaningless marks on paper rather like noughts and crosses; [...]." F.P. Ramsey: Philosophical Papers, Cambridge: (Cambridge University Press 1990), p. 231.

${ }^{10}$ Gödel's theorems and the more generalized case of undecidability will be explored in a future article concerning the nature of a priori knowledge and human intuitions.
} 
Feedback Loop: Significantly, the theorems of number theory are not included as part of the first four criteria. Instead, theorems - like schemas - develop as separate systems of thought within a feedback loop embedded in a mathematical framework. As with schemas, the continuous creation of new theorems often involves trial and error, and also entails the same doubling-up of information and heuristic decision-making over time. At each step in their construction, mathematicians must choose the most appropriate axioms and/or less complex theorems to utilize in the main body of the new theorem. Though new mathematical knowledge (brought about in the a priori forms of human intuition and creativity) plays a major role; the conscious decisions always come down to the same if/then format previously encountered when speaking about schemas. Verbally, in relation to new theorem creation, this format proceeds as follows: "If you see that mathematical statement, then utilize this axiom to manipulate the numbers in this fashion," or "when you have these elements, then utilize this less complex theorem."

The analogy between the theorems of number theory and game schemas can be extended. The feedback loop of the mathematical frame of reference relates the development of new mathematical knowledge in terms of action and reaction over time. Starting from the mathematical frame of reference and moving deeper into the field of number theory, the action/reaction chain of events functions as follows: rudimentary theorems are endowed with mathematical meaning, that is to say, "proven" through their contextual relationships to the mathematical frame of reference. For the set of real numbers, this occurs by the manipulation of the abstract elements (i.e., numbers) through the use of mathematical rules, or "axioms", to prove a proposition (i.e., the objective).

Once a rudimentary theorem is proven, it becomes incorporated into a mathematical action/reaction feedback loop in order to prove new theorems of higher complexity. These more complex theorems in turn are incorporated into still other theorems of even greater complexity. So, simple theorems beget complex theorems, which beget ever more complex theorems and the complexity of mathematical systems increases over time. The leading edges of mathematical knowledge, that is to say, the theorems most distant from the original mathematical framework, become ever more complex and abstract. Outwardly they seem to have little in common with their original frame of reference, but the feedback loop of mathematics remains in operation. Regardless of how abstruse a mathematical theorem eventually becomes, we can understand it only in relation to the simpler axioms and theorems that preceded it and ultimately in relation to the original contextual mathematical framework.

\section{The Physical and Emotional Basis of Human Cognition}

How can it be that mathematics, being after all a product of human thought which is independent of experience, is so admirably appropriate to the objects of reality? ${ }^{11}$

— (Albert Einstein, 1879 - 1955)

Science teaches that cognitive organisms gain knowledge of, and react to, their environment in two ways: by long-term evolutionary adaptation, which results in the development of genetically fixed knowledge structures and behaviours - as previously documented in (Piaget, 1963), (Piaget \& Inhelder, 1967), (Antell \& Keating, 1983), and

\footnotetext{
${ }^{11}$ Geometry and Experience, in: A. Einstein, Ideas and Opinions, (Crown Publishers, 1954), p. 233.
} 
(Wynn, 1992) - and by conscious appraisal and reaction to external stimuli. It is this combination of conscious appraisal and reaction to external stimuli coupled with fixed instinctive knowledge structures and behaviours which is central to this paper.

Writing in regard to (Lakoff \& Núñez, 2000), mathematician and physicist Mario Livio (2002, pp. 250-51) states that, "Mathematics [...] could have originated from a subjective human perception of how nature works. Geometry may simply reflect the human ability to easily recognize lines, edges, and curves. Arithmetic may represent the human aptitude to resolve discrete objects." In this scenario "[...] the mathematics that we have is a feature of the biological details of humans and of how they perceive the cosmos".

A more truncated version of this simple insight is crucial to my argument, as I propose to resurrect but reinterpret the old idea that it is the fundamental character of what we perceive that is most important, not the subjective nature by which we sense and perceive the world around us. For, in the final analysis, the major objects of reality - that is to say, the four macro properties of existence: space, matter, motion, and time, in all their varied forms and nearly infinite combinations and interactions - is all that exists, all that we externally perceive, and all that we can ever directly perceive; all else is just qualia. ${ }^{12}$

It may be that the three-fold mystery as to why various physical conditions seem to have been imposed on us by external reality, why mathematical objects, though imaginary, seem to have real properties, and whether mathematics may be a modified mental model of reality, could be explained by a simple intuitive leap; provided that we surmise that these four properties of existence must themselves be genetically internalized in our neural physiology as the major elemental contextual components of human cognition. How could this happen? How might this subconscious conceptual representation, the one we all utilize a priori to make conscious sense of games and mathematics, be formed - or physiologically internalized-within the brain?

The teleological answer provided by Professor Livio earlier but under different circumstances, is that the major properties of space, matter, motion, and time were all physiologically imposed on life simply because it exists and interacts within this universal environment. We previously discussed how the process that may lead to the creation of an abstract mathematical framework unfolds. Recalling that complex thought may be viewed as a finite regress of less-and-less complex embedded frames of reference with their accompanying schematic reasoning systems; if these less-complex frames of reference are imbued with meaning by the phenomena of a priori thought, one may logically infer that complex thought arises from simpler reasoning systems within less complex frames of reference composed of less-complex a priori knowledge.

If this finite subconscious regress of less complex frames of reference and their accompanying knowledge systems continues to its inevitable conclusion, we arrive at a primal mental representation, the fountainhead of all knowledge. To answer the question as to how this original frame of reference came into being, to determine what structure this first frame of reference possesses, and to draw a strong connection from our abstract contextual mathematical framework down to the primordial mental representation itself, I put forward the following explanation.

\footnotetext{
12 The ontological question as to whether the macro properties of space, matter, motion, and time are indeed the most basic properties of existence may be open to debate, and is not addressed within the scope of this paper.
} 
Long-term evolutionary pressures and changes result in the development of genetically-derived neural networks within the brain. These in turn generate the instinctive behaviours studied by Piaget. From Piaget's genetic epistemological investigations we know that humans are naturally endowed with the basic emotional behaviours needed to survive. Furthermore, this research also indicates that humans are pre-programmed to learn and accumulate information about external existence. I interpret all this to mean that we are not born with a blank slate for a brain, but that we are in possession of an austere a priori frame of reference (i.e., a mental representation of reality) from birth. This first frame of reference is a stripped-down, amorphous representation of the four major properties of existence, plus the original evolutionary genetic causal objective of all life, and - situated within this contextual structure - are the closely associated good-for-life and bad-for-life basic emotional reactions and behaviours that even the lowliest cognitive organisms are born with, and which they automatically express when interacting with their environment.

As we interact with our environment, the genetically internalized frame of reference in conjunction with its embedded positive and negative emotional behaviours - is utilized to order, arrange, and process all incoming information. Over time raw data, transmitted by different forms of external environmental energy, continually streams into our subconscious via our senses. Initially, the brain uses that information to build up the complexity of the primal mental representation with which we are all born.

In subsequent papers on the emergence of meaning and the true nature of a priori knowledge, this complex mapping process will be dealt with in greater detail. For now, it is enough to say that by this process the brain slowly molds what is essentially an inexact representation of existence into closer approximations of the complex external reality all around us. Moreover, this subconscious building-up of previously imposed genetically derived neural networks into more accurate approximations of reality eventually cycles back and heavily influences our conscious perceptual abilities.

Early-on, the brain makes the conscious assumption that space is static and nonmoving. It also assumes, without apologies to Einstein, that space, matter, motion, and time are separate dimensional properties of existence. However, - and it is most likely genetically imprinted - subconsciously the brain combines these four dimensional properties of existence with the positive and negative survival aspects of life's causal objective in order to create a modified spatiotemporal contextual continuum of basic information, within which our more complex reasoning systems develop.

Once this modified mental model of reality is sufficiently built-up, the brain thereafter uses any new information to orient and guide our bodies through space in relation to our previously built frame of reference. It also utilizes new incoming information as part of the appraisal phase, distinguishing and mapping the position of any perceived objects, i.e., matter that occupy the space around us. It tracks the motion of the most important objects relative to space and time and updates any positive or negative (i.e., good or bad for life) connotations of the moving objects and/or the end result of their motion.

Provided that the basic data has been categorized correctly the brain embarks on a proper course of action, by accessing the most appropriate schemata, based on a close correspondence between our internal frames of reference and the incoming information. 
From this generalized introspection it may be supposed that the external properties of space, matter, motion, and time, plus the internal causal objective of all life come together to subconsciously form the contextual framework that we all possess and utilize to comprehend the complex web of reality all around us. ${ }^{13}$ This occurs precisely because these four macro properties of the cosmos, by their mere existence, convey information and routinely impart this information about their state of being onto our developing neural physiology. The same may be said of the "fifth element" of all games, the "objective." Research suggests that the original evolutionary objective of all life survival - is automatically genetically imprinted on complex living organisms as they struggle to exist and proliferate within an ever-changing and often hostile environment.

In his book, The Conscious Mind, David Chalmers puts forward the proposition that conscious experience should be treated as a real property of the natural world (1996, pp. 127-128). As such, consciousness must be considered an irreducible essence, akin to the four dimensional properties of space, matter, motion, and time. I agree, with his elevation of the importance of conscious perception, but with provisos: perhaps this accolade should more appropriately be accorded to our innate causal objective and its closely associated positive and negative emotions, and not consciousness per se (See also footnote 13).

Obviously, "we have bodies that move in time and space, senses that have a field-like nature, and experiences of the divisibility of substance (objects, etc.) that produce the lowest level, grounded qualia of experience". 14 However, at the most basic level of awareness, we tend to lose sight of the fact that raw sensory information cannot be perceived until after we subconsciously interact with it. We are constantly bombarded with a great deal of sensory data, but only the timeliest and most important information makes it past the thalamus to be consciously considered. The initial subconscious physiological progression by which the brain receives, orders, and arranges unfiltered data then selectively brings only the most vital processed perceptual information into conscious focus - forces upon us a continuous series of choices, with each choice being the most appropriate course of action in a given situation or frame of reference at that moment. Clearly, such choices cannot be undertaken without the primal causal objective automatically being part of the subconscious physiological process by which the brain categorizes and weighs the importance of all incoming information. Such a criterion would logically be necessary in order for the brain to take immediate action, whether subconsciously or consciously, when confronted with any external situation.

\footnotetext{
${ }^{13}$ The process by which we learn to manipulate and interpret information and which results in the eventual development of consciousness does not start at this point. It begins with the far simpler synchronous firing of related neurons and gains in complexity over time. However, at some stage in this process the brain binds the most important information into a single whole; that is to say, contextual conninuuam. I believe this construct to be based on the naturally occurring representational scheme we perceive plus the causal objective of all life, and the embedded emotional valuations we attach to all higher order schematic information.

${ }^{14}$ Grand, Steven. (2006): ‘Re: General Information’ E-mail to author.
} 
Furthermore, of the five basic contextual components of our primal frame of reference, i.e., space, time, matter, motion, and our causal objective - the original evolutionary survival objective of all life seems to be the major driving force behind the generation of our most primitive emotions. Moreover, within the feedback loop our predominant consciously expressed emotional reactions to external circumstances, also seem to be the most likely and necessary factors involved in the eventual generation and development of "cognitive relevance" in both the restrictive sense of the phrase and the more general sense, i.e., "meaning." In order to stay alive and healthy and to avoid injury or death, all complex organisms naturally evolved the capacity to identify, during the appraisal phase, those factors most important to their ongoing existence. In accordance with their assessment of a given situation, they "emotionally" act; in other words, they initiate the classic fight-or-flight response so as to maximize their chances of staying alive and healthy. I submit that, in humans, this emotive reaction to the external factors affecting our immediate survival has further evolved into the conscious capacity to weigh the positive and negative causal relevance of any piece of information, which on an abstract level may no longer have any emotionally-perceived survival value.

The idea that our genetically-endowed emotions play a vital and necessary part in the development of "cognitive relevance" and in the grounding of conscious "meaning" may be inferred from the scientific literature. The research of neurobiologist Antonio Damasio is a good case in point. Professor Damasio's empirical studies have appeared in scientific journals around the world. He has also written a series of less-technical books for the lay public. These include Looking for Spinoza, The Feeling of What Happens, and Descartes' Error. In his book, Looking for Spinoza, Dr. Damasio's position is that emotions and feeling evolved out of the complex organic mechanisms that subconsciously monitor and regulate the internal body state. That is to say, they emerged from the physiological processes that mediate our internal response to external circumstances, and by doing so, solve the most critical problems of survival (2003, pp. 27-42). He uses the term "homeostasis" for this physiological monitoring.

While positive and negative feedback is the main component of the learning process, my position is that emotions and feeling are not required at the Hebbian level. ${ }^{15}$ They do, however, consciously bridge the gap between external situations and our internal body state. In other words, emotions and feeling weigh or internally compare what we've learned or experienced in the past to current external circumstances at the higher-order schematic level. ${ }^{16}$ This very small change in relation to the subconscious physiological monitoring documented by professor Damasio is consistent with standard evolutionary theory, and very much in line with his original thesis. For in his earlier book, Descartes' Error, Damasio clearly states that "Emotions and feeling, along with the covert physiological machinery underlying them, assist us with the daunting task of predicting an uncertain future and planning our actions accordingly." (1994, p. xiii)

\footnotetext{
${ }^{15}$ The Organization of Behaviour (John Wiley \& Sons, New York, 1949).

${ }^{16}$ With that in mind, I believe that emotions originally evolved as an aid to memory creation, memory retention, and recall.
} 
According to Professor Damasio, emotions and feeling assist us now in predicting the future and planning our actions. However, at the time they initially made their appearance, emotions and feeling most likely were the first and second types of conscious reactions in response to external situations that emerged. All the conscious logic and rationality that we now use for that purpose evolved later. They evolved out of emotions and feeling, and in consequence, retained the same function. Our conscious ability to react to external circumstances in relation to past experiences simply became more complex, abstract, consistent, and as a result less irrational over time.

\subsection{Invariant Iterated Re-Expression}

In each of his books, Dr. Damasio and his team of researchers provide evidential studies attesting that physical damage to the emotional centers of the brain, primarily the amygdala, seriously affects the proper development and expression of critical thinking. Why do injuries to the amygdala and other emotional centers of the brain eventually derail the proper growth and development of conscious thought? To my mind, such findings indicate that impairment to the emotional centers of the brain can have such demonstrable affects, only if the primal causal objective of all life and its consciously expressed elemental emotions are essential components of the underlying physiological contextual structure that engenders our schematic reasoning abilities.

In essence, within the feedback loop, our primitive emotional reactions to external stimuli physiologically combine with abstract conceptualizations of space, matter, motion and time, plus the original evolutionary causal objective, to form a modified spatiotemporal continuum of basic information in which complex thoughts, i.e., schemas coalesce. ${ }^{17}$ Once life arrived at the best holistic representation with which to appropriately act and react to the vagaries of external existence, the resultant complete frame of reference with its five fundamental properties and time was never radically altered. As exemplified by the contextual structure of number theory and the analogues multitude of games with their different interpretations of the same external pattern, the expression (i.e., the perceptual characteristics) of each property may be incrementally modified, or more specially, extended over time in order to generate more enhanced frames of reference. The new frames of reference better model existing characteristics of reality and in the process produce increasingly abstract and ever more precise and consistent reasoning systems. But the singular essence of each core component never changes; in other words, the underlying primal attribute of each criterion remains invariant. The initial frame of reference is simply refined, re-expressed, and repeated over and over again, in an open-ended iterated cycle of creative discovery.

The reader will note that what I have heretofore described in the common parlance as the complete frame of reference of a game may also be understood in more technically precise mathematical terminology as a chaotic system. As with all chaotic systems, small

\footnotetext{
${ }^{17}$ My inductive investigation concerning the contextual emergence of mathematics is consistent with the empirical studies of the neuroscientists Otto Creutzfeldt, (Creutzfeldt, 1977, pp. 507-517) and Vernon Mountcastle, (Mountcastle, MIT Press, 1978, pp. 7-50). For both researchers contend that a common spatiotemporal pattern recognition algorithm seems to be functioning everywhere throughout both hemispheres of the neocortex.
} 
changes to any of the initial conditions of our cognitive framework dramatically alter the conscious expression of the developing feedback loop, i.e., the schematic body of the system. This may turn out to be the rationale for the immense complexity of human mind. It could also explain why mathematics is so much more than just symbolic word problems expressed through sentences, and why deductive thought outwardly seems quite unrelated to empirical reasoning. But underlying all the diverse complexity and perceived differences is the same modified spatiotemporal pattern and order.

In a fashion quite analogous to game-playing - during the appraisal phase which always varies depending on the exact conditions - the brain finds survival benefits in being able to accurately predict and make use of favorable external circumstances, or to detect and avoid dangerous situations before they become a problem. Therefore, within the feedback loop, the brain strategically devises a hierarchical system of contextual thought - initially genetic emotional behaviours, but thereafter based on incremental modifications to the five basic properties of our initial frame of reference.

These small changes to the primal conceptual framework result in massive modifications to the original embodied feedback loop of consciously expressed emotional reactions in response to past experiences. The resultant vastly more complex reasoning systems allow us to fine tune the intricate thought patterns necessary to more efficiently take advantage of opportunities, and/or to better perceive and more subtly and economically react to threats or overcome them before they become a serious concern.

Emotions, genetically designed by evolution to solve the most fundamental problems of survival, and at all times available to be utilized during life-threatening and other stressful situations, also serve as the conscious basis of human cognition. Through successive hierarchical permutations of the primal frame of reference, our conscious emotional reactions to external stimuli are reformulated within new schematic systems; with the lower less evolved schematic systems forming the basis for the new more enhanced frames of reference and their associated reasoning systems that follow. Initially, the raw emotions bequeathed to us at birth are modified and moderated, resulting in the emergence of feeling. In turn, our inexact feelings of what happens are further transformed into more concrete conceptualizations. Throughout this transformation process the emotional impact is diluted, but our capacity to be aware of external circumstances in relation to our internal body state, i.e., what we have previously learned or experienced, is maintained. It just becomes more complex, abstract, and consistent over time, resulting in the eventual embodiment of visceral meaning, language, logic, and finally the development of symbolic mathematics and all abstract thought. ${ }^{18}$

\footnotetext{
${ }^{18}$ By chaotically linking the emergence of mathematics to massive changes in our feedback loop of consciously perceived emotional reactions in response to incremental changes to the five basic categories of our primal frame of reference, we may have also taken the first tentative steps towards unraveling another troublesome problem of consciousness: the problem of intrinsic meaning. In his influential paper, The Symbol Grounding Problem, Stevan Harnad recounts two variations of a single very important question. First, "How can the semantic interpretation of a formal system be made intrinsic to the system [...]." In other words, "How can the meanings of the meaningless symbol tokens manipulated solely on the basis of their (arbitrary) shapes be grounded in anything but other meaningless symbols?" Harnad proceeds to sketch-out a possible candidate solution that is remarkably similar, in its initial articulation, to the one presented in this paper. According to him, "Symbolic representations must be grounded bottom-up in nonsymbolic representations of two kinds: (1) "iconic representations", which are analogs to proximal sensory projections of distal objects and events, and (2) "categorical representations", which are learned
} 


\section{Conclusion}

I should attempt to treat human vice and folly geometrically, [...]. The passions of hatred, anger, envy and so on, considered in themselves, follow from the same necessity and efficacy of nature; [...]. I shall, therefore, treat the nature and strength of human emotions, actions, $[\ldots]$ and desires in exactly the same manner as though I were concerned with lines, planes, and solids. $^{19} \quad$ - (Benedictus De Spinoza, $1632-1677$ )

From the time of Renee Descartes $(1596$ - 1650) to the present day, it has generally been assumed that there is a dichotomy of consciousness; that there exists a deep division between our emotions and feelings and all abstract thought. However, my analysis leads to the conclusion that this distinction between the higher-level experienced dominated frames of reference that engender intrinsic meaning, language, logic, mathematics and science - and the lower-level genetically based perceptual frames of reference that generate our conscious emotions and feelings - is not so much a difference in kind, as Descartes believed, but rather a difference in the degree or level of complexity.

Therefore, it is inductively proposed that all higher order epistemological complexities of abstract thought and knowledge, from the subtlest nuance of conscious meaning and language to the most abstruse mathematical theorems and scientific theories, emerge out of a continually evolving contextual feedback loop or loops of ever-more complex experiences derived from all the threats and opportunities that cognitive organisms contend with. The initial feedback loop starts with our primitive emotional reactions to external stimuli. It then gains in complexity over time through bifurcation; that is to say, through the branching off into several new feedback loops embedded within multiple and more complex hierarchical subconscious frames of reference.

I also submit that, regardless of the degree of complexity, each individual frame of reference, i.e., chaotic system is in its own way an iterated permutation of the original primordial framework. The idea that higher order conscious thought manifests itself as contextual schemata within a series of hierarchical subconscious frames of reference, and that each frame of reference is in its own way a chaotic representation of reality, with its own version of a static field, may be the most difficult facet of my dissertation, for we are not consciously aware that this is indeed the case. Nevertheless, in future papers I intend to support the various concepts and conclusions presented here by providing detailed frame-based explanations for several key problems of human cognition. These include:

and innate feature-detectors that pick out invariant features of objects and event categories from their sensory projections." (1990, p. 335).

${ }^{19}$ Preface to Part III, Of the Origin and Nature of Affects, in: Ethics Demonstrated in Geometric Order and Divided into Five Parts (Amsterdam, 1677) in: On the Improvement of the Understanding; The Ethics Correspondence (Dover Publications, 1955), p. 129. 
- A historical review of mathematical consistency; to include a more thorough and comprehensive examination of the subjective/objective nature of conscious thought.

- $\quad$ A frames-based reassessment of Russell's paradox, the Barber paradox, and a general theoretical analysis of the physiological generation of antinomies.

- An investigation into the contextual structure underlying language and the emergence of abstract meaning.

- A frames-based description of a priori knowledge, including an examination of the mysterious human faculties of intuition, epiphany, and creativity, with a more detailed analysis of the chaotic structure of complex thought.

The outline of consciousness that I propose here provides a single reasonable solution to the Frame(s) Problem, to the mysterious effectiveness of mathematics in the natural sciences when describing the workings of the cosmos, and possibly also to the symbol grounding problem. However, the picture is still incomplete. For I have neither precisely detailed the true nature of a priori knowledge, nor have I sufficiently explained why and how higher-level conscious perceptions such as language, logic, and mathematics emerge out of this primordial contextual framework. Additionally, in its present embryonic state, the explanation puts me at odds with the current pronouncements of some linguists, psychologists, and other cognitive researchers - scientists such as George Lakoff and Rafael E. Núñez, for example. Lakoff and Núñez (2000, pp. 337-79) argue that our system of deductive thought may have come about as a consequence of the evolutionary and biological details plus the cultural history of humans, and how we subjectively sense and process incoming external information. Such broad a stance is correct but only to a point, for - if unchecked - it leads to the natural supposition that other descriptions of nature different from our mathematics may exist, and that an intelligent extraterrestrial civilization with dissimilar mechanisms of perception could develop a different mathematics than our own (Livio, 2002, p. 251).

This view seems plausible initially, for it does have a basis in actual fact. Terrestrial animals' perception of reality differs from human perception. Bats, whales, and dolphins, for example, easily hear high pitched sounds in a frequency range well above our ability to detect. Many insects and birds have the ability to see in the ultraviolet photoelectric spectrum, a frequency of radiant energy above our visual range. Homing pigeons have the capacity to detect electromagnetic lines of force. Similarly, sharks and other species of fish perceive weak electromagnetic fields. These are senses that humans do not possess. 
Undeniably, terrestrial animals experience reality differently than we do, but their perceptual abilities still detect only some combination of space, matter, motion and time. As previously noted, I maintain that human mathematics contextually developed out of a hierarchical chaotic formulation of these four dimensional properties of the cosmos, plus the genetically-derived causal objective, combined and integrated within a continuously evolving feedback loop originally consisting of our most primitive emotions. These macro properties of existence make up four-sixths of our complete contextual frame of reference. As there is no place in the observable universe where these major properties do not hold sway, regardless of the degree to which any living thing perceives its external environment, at the lowest level all complex organisms still basically only perceive space, matter, motion, and time. Therefore, any life form sufficiently intelligent to have evolved a system of abstract mathematics based on its own sensations and perceptions, on Earth or elsewhere, would necessarily develop a system of deductive thought similar to our own. 


\section{References}

Antell, S. E., Keating, D. P. (1983): "Perception of Numerical Invariance in Neonates", Child Development, 54: pp.695-701.

Atmanspacher, H. (2007): "Contextual Emergence from Physics to Cognitive Neuroscience”, Journal of Consciousness Studies 14(1/2), pp. 18-36.

Atmanspacher, H., Graben, P. C. (2007): “Contextual Emergence of Mental States from Neurodynamics”, Chaos and Complexity Letters, Vol. 2, Num 2/3, pp. 151-168.

Bishop, R. C., and Atmanspacher, H. (2006): "Contextual Emergence in the Description of Properties", Foundations of Physics, Vol. 36. No. 12 pp. 1753-77.

Chalmers, D. J. (1996): The Conscious Mind: In Search of a Fundamental Theory, Oxford: Oxford University Press.

Creutzfeldt, O. D. (1977): "Generality of the Functional Structure of the Neocortex," Naturwissenschaften, Vol. 64 pp. 507-517.

Damasio, A. R. (1994): Descartes' Error: Emotion, Reason, and the Human Brain, G. P. Putnam's Sons, Putnam Berkley group Inc. New York, New York.

. (1999): The Feeling of What Happens: Body and Emotion in the Making of Consciousness, Harcourt Inc. New York, New York. . (2003): Looking for Spinoza: Joy, Sorrow, and the Feeling Brain, Harcourt Inc. New York, New York.

Einstein, A. (1954): “Geometry and Experience," in: A. Einstein, Ideas and Opinions, Crown Publishers, Inc. New York, p. 233.

Galilei, G. (1623): Il Saggiatore, 'The Assayer,' Tans. Stillman Drake and C.D.O’Malley, in: The Controversy of the Comets of 1618, (1960): University of Pennsylvania Press. pp. 151-336.

Harnad, S. (1990): “The Symbol Grounding Problem”, Physica D 42: pp. 335-346.

Hebb, D. O. (1949): The Organization of Behaviour: John Wiley \& Sons, New York.

Hilbert, D. (1900): 'Mathematical Problems,' Trans. Mary Winton Newson, in: Mathematical Developments Arising from Hilbert Problems, (1976): Edited by Felix E. Browder, American Mathematical Society, Vol. 28, pp.1-34.

Kant, I. (1783): Prolegomena to Any Future Metaphysics translated and edited by Gary Hatfield, Cambridge Texts in The History of Philosophy, (2004): Cambridge University Press. 
(1787): Critique of Pure Reason, second ed. Trans. Norman Kemp Smith, with a new preface by Howard Caygill, (2003): London: Palgrave Macmillan Publishers.

Lakoff, G., Núñez, R. E. (2000): Where Mathematics Comes From: How the Embodied Mind Brings Mathematics into Being, New York: Basic Books.

Livio, M. (2002): The Golden Ratio: The Story of Phi. The World's Most Astonishing Number, New York, New York: Random House.

Mountcastle, V. B. (1978): “An Organizing Principle for Cerebral Function: The Unit Model and Distributed System," in Gerald M. Edelman and Vernon B. Mountcastle, eds., The Mindful Brain, Cambridge, Mass., MIT Press. pp. 7-50.

Nadeau, R., Kafatos, M. (2001): THE NON-LOCAL UNIVERSE: The New Physics and Matters of the Mind, New York: Oxford University Press.

Piaget, J. The Origins of Intelligence in Children, (1963): W. W. Norton \& Company, Inc., New York, New York.

Piaget, J., Inhelder, B. (1967): The Child's Conception of Space, W. W. Norton \& Company, Inc., New York, New York.

Ramsey, F.P. (1926): “Mathematical Logic,” F.P. Ramsey: Philosophical Papers, (1990): edited by D.H. Mellor, Cambridge: Cambridge University Press. pp. 225-244.

Spinoza, B. De. (1677): "Part III, Of the Origin and Nature of Affects," in: Ethics Demonstrated in Geometric Order and Divided into Five Parts, Trans. R.H.M. Elwes, in: On the Improvement of the Understanding; The Ethics Correspondence, (1955): Dover Publications, pp. 128-186.

Wigner, E. P. (1960): "The Unreasonable Effectiveness of Mathematics in the Natural Sciences", Communications in Pure and Applied Mathematics, Vol.13, pp. 1-14.

Wynn, K. (1992): “Addition and Subtraction by Human Infants”, Nature, 358, pp. 749-750. 